\title{
Understanding computer-mediated communication attributes and life satisfaction from the perspectives of uses and gratifications and self-determination
}

\begin{abstract}
Based on the theories of uses and gratifications, and self-determination, we examined a model linking computer-mediated communication (CMC) attributes to psychological need satisfaction in online friendships and to life satisfaction in a sample of school-aged adolescents $(\mathrm{N}=1572)$. Our findings suggest direct links between media orientations (i.e., attitude toward online relationship formation and Internet habit strength) and psychological need satisfaction in online friendships. We also reported direct links between online communication, online self-disclosure and psychological need satisfaction in online friendships, and also a direct link between psychological need satisfaction in online friendships and life satisfaction. Despite these direct links, online communication and online self-disclosure significantly mediated the link between attitude toward online relationship formation and psychological need satisfaction in online friendships. In this pattern of links, both mediators were equally strong. Online communication and online self-disclosure also significantly mediated the link between Internet habit strength and psychological need satisfaction in online friendships. For this pattern of links, both mediators differed significantly in strength. Online communication emerged as a stronger mediator than online self-disclosure. Our findings suggest that CMC attributes may serve as a new social milieu for adolescent subjective well-being.
\end{abstract}

Keyword: Life satisfaction; Online friendships; Psychological need satisfaction; Selfdetermination theory; Uses and gratifications theory 